\title{
Continuing efforts to standardize measured serum growth hormone values in Japan
}

\author{
Noriyuki Katsumata, Akira Shimatsu, Katsuhiko Tachibana, Naomi Hizuka, Reiko Horikawa, \\ Susumu Yokoya, Ke-ita Tatsumi, Takahiro Mochizuki, Makoto Anzo and Toshiaki Tanaka
}

The Study Committee for GH and Its Related Factors, The Foundation for Growth Science, Tokyo, Japan

\begin{abstract}
Determination of serum growth hormone (GH) levels is mandatory for diagnosis of GH deficiency and excess. In the present study, we, the Study Committee for GH and Its Related Factors, The Foundation for Growth Science, Japan measured GH values in serum samples using all the commercially available kits in Japan. Significant discrepancies in the GH values were observed among the kits in spite of using the unified recombinant human GH-based standards. To deal with the discrepancies, we established a formula using a linear structural relationship model and were able to standardize the GH values. We propose to use the formula to diagnose GH deficiency and excess in Japan.
\end{abstract}

Key words: Growth hormone (GH), Immunoassay, Assay kit, WHO IS, Somatropin

DETERMINATION of growth hormone $(\mathrm{GH})$ levels in serum samples is essential for diagnosis of $\mathrm{GH}$ deficiency and excess [1-3]. In clinical practice, GH levels have been determined by immunological methods including radioimmunoassay and enzyme immunoassay. In Japan, several reagents to measure serum GH (GH assay kits) have been commercially available. Until 2005, measured GH values in individual sera varied significantly among GH assay kits. To deal with the variations, we as the Study Committee for GH and Its Related Factors, The Foundation for Growth Science, Japan collaborated with the Study Group for Hypothalamo-Pituitary Disorders of the Ministry of Health, Labour and Welfare (MHLW), Japan and the suppliers of GH assay kits, and provided the formulas to adjust the measured values to their average. Since the majority of discrepancies in the measured values were attributed to the unequal potencies of the in-house GH standards of the suppliers, the unified recombinant human GH (rhGH)-based standards were introduced to all GH assay kits in 2005 [4-6]. As the result, the discrepancies in measured values among them became

Submitted Apr. 20, 2016; Accepted Jul. 4, 2016 as EJ16-0198 Released online in J-STAGE as advance publication Jul. 22, 2016 Correspondence to: Noriyuki Katsumata, M.D., Ph.D., Department of Molecular Endocrinology, National Research Institute for Child Health and Development, 2-10-1 Okura, Setagaya-ku, Tokyo 1578535, Japan. E-mail: katsumata-n@ncchd.go.jp less prominent, and the cutoff value in the GH stimulation tests was set to $6 \mathrm{ng} / \mathrm{mL}$ rather than previous 10 $\mathrm{ng} / \mathrm{mL}$ since the measured $\mathrm{GH}$ values became $60 \%$ of those obtained before the introduction of rhGH-based standards [5]. Recently, however, the considerable variations were noticed again by the annual nationwide control survey for immunoassays [7-10], so that additional studies became required. Here, we describe the results of the recent studies.

\section{Materials and Methods}

Serum samples were collected after informed consent was obtained from each subject and were kept at $-70{ }^{\circ} \mathrm{C}$. Approval by the Institutional Review Boards was acquired when necessary. $2^{\text {nd }}$ WHO IS somatropin (recombinant human GH) (code: 98/574) was purchased from the National Institute for Biological Standards and Control (NIBSC). GH assay kits used in this study are shown in Table 1.

In 2012, we determined GH levels in 100 sera from healthy adult volunteers using $5 \mathrm{GH}$ assay kits listed in Table 1. To standardize measured values, equations were calculated using a linear structural relationship model as reported previously [6]. In addition, we serially diluted the IS somatropin (NIBSC 98/574) solution with the phosphate buffered saline and determined GH content in the diluted materials using the TFB, Roche 
Table 1 GH assay kits available in Japan

\begin{tabular}{lll}
\hline GH assay kit (abbreviations) & $\begin{array}{l}\text { Assay } \\
\text { method }\end{array}$ & Supplier \\
\hline GH Kit Daiichi (TFB) & IRMA & TFB, Inc. \\
\hline EClusys hGH (Roche) & ECLIA & Roche Diagnostics, Inc. \\
Access hGH (Beckman) & CLEIA & Beckman Coulter, Inc. \\
IMMULYZE GH II (Siemens) & CLEIA & Siemens HCD, Inc. \\
E Test TOSOH II HGH (Tosoh) & IEMA & Tosoh Co., Ltd. \\
\hline IRMA, immunoradiometric assay; & \\
ECLIA, electrochemiluminescence immunoassay; \\
CLEIA, chemiluminescent enzyme immunoassay; \\
IEMA, immunoenzymometric assay.
\end{tabular}

and Beckman kits. Data of the diluted IS somatropin solution were analyzed with a linear regression model.

In 2015, since the TFB kit was no longer available, we determined GH levels in 103 sera using the $4 \mathrm{GH}$ assay kits other than the TFB kit. Forty sera were the stored samples since 2012, and the remaining 63 were newly collected from short children. To validate the formula established in 2012, we calculated equations using the data transformed with this formula and the linear structural relationship model.

\section{Results}

\section{Study in 2012}

In Fig. 1 measured GH levels in individual sera with the $5 \mathrm{GH}$ assay kits were plotted against their averages. Their correlation between the kits was acceptable, but significant discrepancies in the slopes were observed among the kits. The TFB kit tended to give higher values, while the Beckman kit lower values. To adjust the measured values to their average, equations were calculated using a linear structural relationship model (Table 2).

In Fig. 2 measured GH content in the diluted IS somatropin solution with the TFB, Roche and Beckman kits is plotted against the expected values from the dilution factors. The measured content was in good agreement with the expected values.

Based on these results, the following formula was recommended: $\mathrm{Y}=1.4 \mathrm{X}$. Here, $\mathrm{Y}$ denotes standardized $\mathrm{GH}$ values, and $\mathrm{X}$ denotes measured $\mathrm{GH}$ values when the Beckman kit is used.

\section{Study in 2015}

In Fig. 3 measured GH levels with the $4 \mathrm{GH}$ assay kits were plotted against their averages. The Beckman

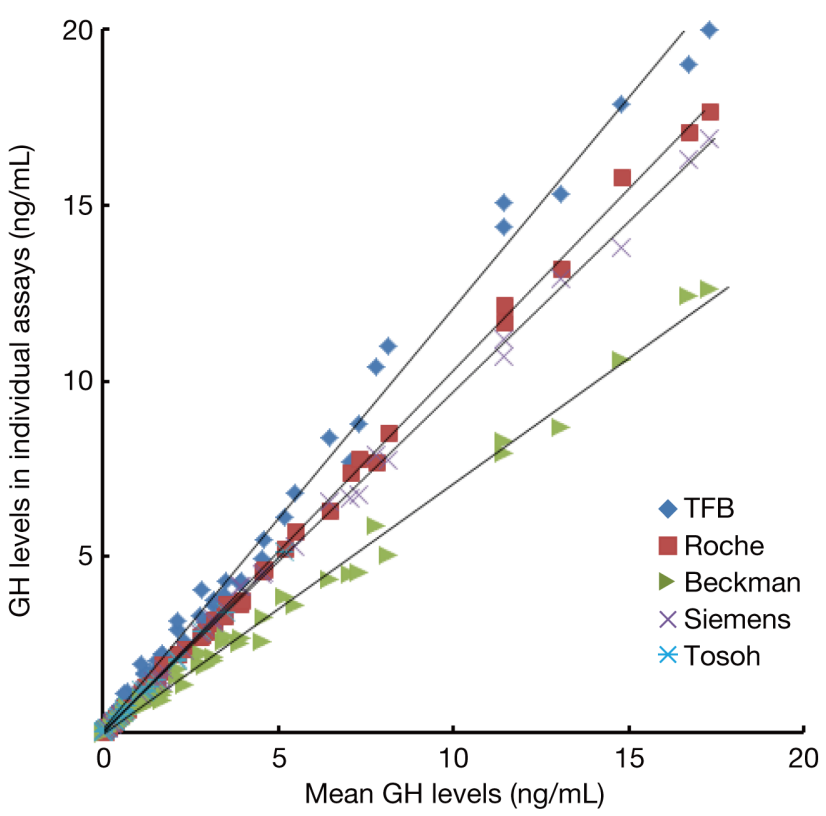

Fig. 1 Relationship between mean and individual GH values measured by commercially available kits in the year 2012 .

Table 2 Equations calculated using a linear structural relationship model to adjust the measured GH values (X) in 2012 to their averages (Y)

\begin{tabular}{lcc}
\hline GH assay kit & Equation & Correlation coefficient \\
\hline TFB & $\mathrm{Y}=0.83 \mathrm{X}-0.06$ & 0.997 \\
Roche & $\mathrm{Y}=0.97 \mathrm{X}+0.03$ & 0.999 \\
Beckman & $\mathrm{Y}=1.41 \mathrm{X}+0.07$ & 0.997 \\
Siemens & $\mathrm{Y}=1.03 \mathrm{X}-0.04$ & 0.999 \\
Tosoh & $\mathrm{Y}=1.01 \mathrm{X}$ & 0.999 \\
\hline
\end{tabular}

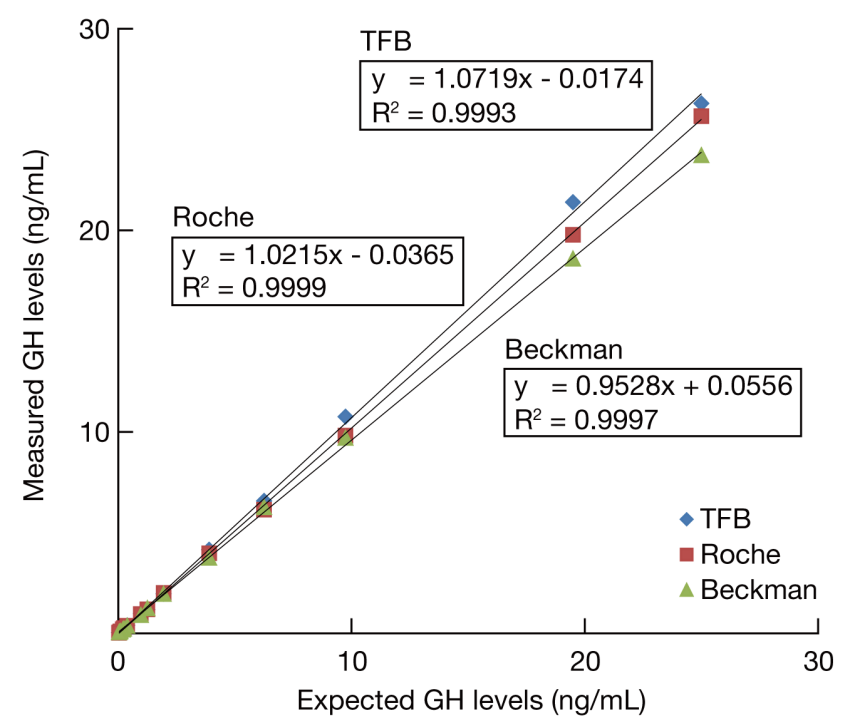

Fig. 2 Relationship between the expected and measured GH values in serially diluted IS somatropin solutions. 
kit tended to result in lower values. To verify the formula established in 2012, we transformed measured $\mathrm{GH}$ values with this formula. In Fig. 4, transformed GH values were plotted against their averages. Equations were calculated using the transformed data and the linear structural relationship model (Table 3). The same formula as that established in 2012 was recommended.

\section{Discussion}

In the present studies, we measured GH levels using commercially available GH kits in Japan, and observed discrepancies in the measured values among the kits. To evaluate the potencies of GH standards in the kits, we quantified GH content in the diluted IS somatropin solutions using the kits. The obtained content was the same as the expected values. Therefore, it is concluded that the potencies of GH standards in the kits are equivalent and that the discrepancies in the measured values are due to other causes than inequality of the standards.

Immunoassays for $\mathrm{GH}$ inherently cannot avoid some variability in the determined values because of the molecular heterogeneity in circulating $\mathrm{GH}$, the presence of circulating $\mathrm{GH}$ binding protein and matrix effects $[11,12]$. Therefore, it is preferable to establish reference ranges and cut off values in GH stimulation and suppression tests for each assay. However, such an ideal approach is practically impossible especially in children. One of the practical approaches is to standardize the values determined by various GH assay kits as we have performed.

In Japan, almost all the cost for GH treatment of children with GH deficiency is supported by social insurance and by a grant for special chronic children's disease from MHWL [4]. Nowadays, the cost for GH treatment of adults with severe GH deficiency is also supported in part by a grant for intractable diseases from MHWL. Thus, nationwide criteria for diagnosis of GH deficiency need to be established. The Study Group for Hypothalamo-Pituitary Disorders of MHWL has been making efforts to establish the criteria, including cutoff values in GH stimulation tests. To equalize interpretations of individual GH stimulation tests, we have been working on formulas to standardize $\mathrm{GH}$ values measured by different $\mathrm{GH}$ assay kits. We have proposed a new formula in 2012, which is approved by MHLW. In 2015, we have reconfirmed that the formula is still appropriate. Therefore, we propose to

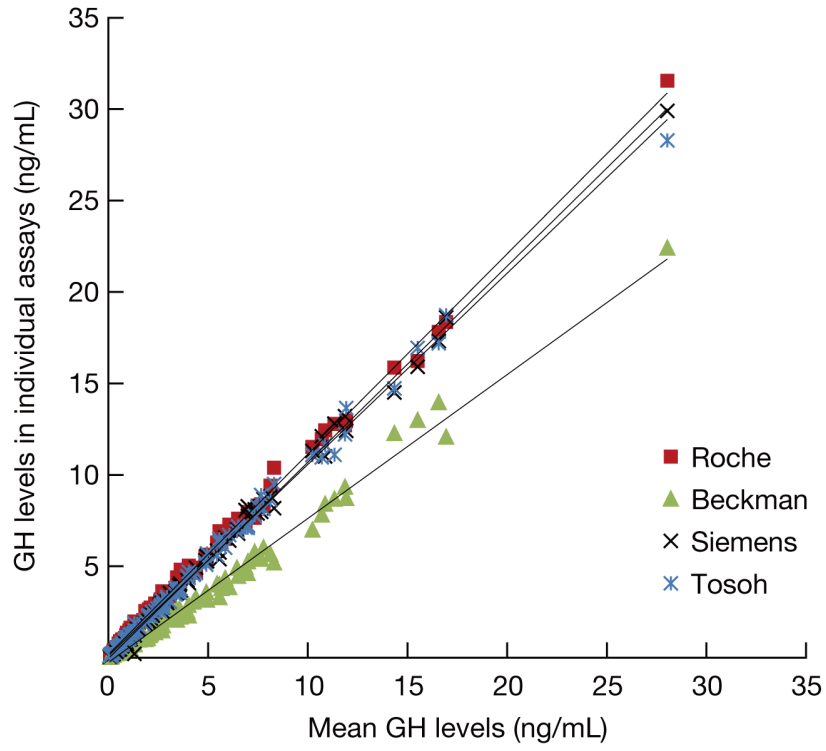

Fig. 3 Relationship between mean and individual GH values measured by commercially available kits in the year 2015 .

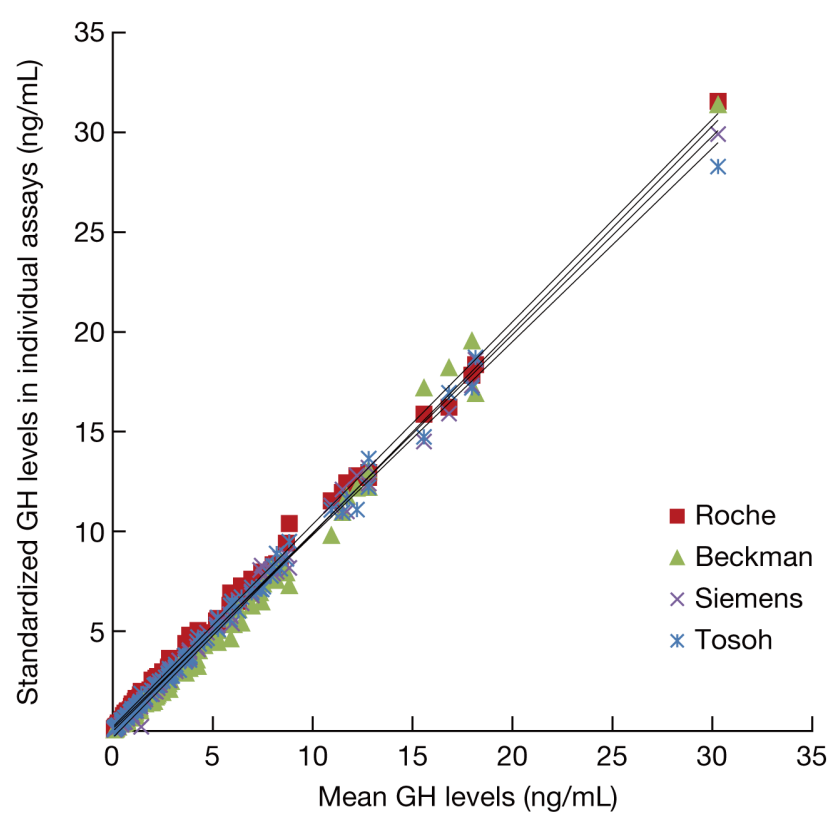

Fig. 4 Relationship between mean and standardized GH values measured in the year 2015 .

Table 3 Equations calculated using a linear structural relationship model to adjust the transformed $\mathrm{GH}$ values (X) in 2015 to their averages (Y)

\begin{tabular}{lcc}
\hline GH assay kit & Equation & Correlation coefficient \\
\hline Roche & $\mathrm{Y}=0.98 \mathrm{X}-0.20$ & 0.997 \\
Beckman & $\mathrm{Y}=0.97 \mathrm{X}+0.33$ & 0.994 \\
Siemens & $\mathrm{Y}=1.00 \mathrm{X}+0.02$ & 0.997 \\
Tosoh & $\mathrm{Y}=1.03 \mathrm{X}-0.12$ & 0.997 \\
\hline
\end{tabular}


keep using the same formula in the meanwhile. It is noteworthy that some of the Y-interceptions are apart from 0 in the equations of 2015 (Table 3). Therefore, we should pay attentions when we interpret the standardized values in the lower range of GH.

Internationally, Clemmons has published the consensus statement on the standardization and evaluation of GH assays [12]. Collaborators have been working on the harmonization of GH measurements, which might include identification of a commutable matrix reference material and evaluation of liquid chromatography mass spectrometry as a reference measurement procedure [13].

In conclusion, we have established a practical formula using a linear structural relationship model to standardize the measured GH values. Further efforts are needed to harmonize GH measurements.

\section{Acknowledgements}

This study is supported in part by a Grant for Research on GH and Its Related Factors from The Foundation for Growth Science, Japan and a Grant for Research on Intractable Disease from MHLW. We are grateful to Dr. Tomoko Suzuki (Special Reference Laboratories, Inc., Japan), Mr. Hiroo Yamamoto (TFB, Inc., Japan), Dr. Mikako Hiura (Roche Diagnostics, Inc., Japan), Ms. Kazuko Nishimura (Beckman Coulter, Inc., Japan), Mr. Tatsuo Funakoshi (Siemens HCD, Inc., Japan), and Mr. Koji Maki (Tosoh Co., Ltd., Japan) for their assistance.

\section{Disclosures}

None of the authors have any conflicts of interest associated with this research.

\section{References}

1. Growth Hormone Research Society (2000) Consensus guidelines for the diagnosis and treatment of growth hormone $(\mathrm{GH})$ deficiency in childhood and adolescence: summary statement of the GH Research Society. J Clin Endocrinol Metab 85: 3990-3993.

2. Growth Hormone Research Society; Pituitary Society (2004) Biochemical assessment and long-term monitoring in patients with acromegaly: statement from a joint consensus conference of the Growth Hormone Research Society and the Pituitary Society. J Clin Endocrinol Metab 89: 3099-3102.

3. Ho KK; 2007 GH Deficiency Consensus Workshop Participants (2007) Consensus guidelines for the diagnosis and treatment of adults with GH deficiency II: a statement of the GH Research Society in association with the European Society for Pediatric Endocrinology, Lawson Wilkins Society, European Society of Endocrinology, Japan Endocrine Society, and Endocrine Society of Australia. Eur J Endocrinol 157: 695-700.

4. Tanaka T, Takano K, Hanew K, Nishi Y, Igarashi Y, et al. (1998) Registration system for growth hormone (GH) treatment with standardized immunoreactive GH values in Japan. Endocr J 45: 459-465.

5. Tanaka T, Tachibana K, Shimatsu A, Katsumata N, Tsushima T, et al. (2005) A nationwide attempt to standardize growth hormone assays. Horm Res 64 (Suppl 2): 6-11.

6. Saito T, Tachibana K, Shimatsu A, Katsumata N, Hizuka $\mathrm{N}$, et al. (2006) Standardization of blood growth hormone levels measured by different kits using a linear structural relationship. Clin Pediatr Endocrinol 15: 79-84.

7. Subcommittee for Radioisotope in vitro Test, Medical Science and Pharmaceutical Committee, Japan
Radioisotope Association, Immunoassay Research Society Japan (2006) A summary report on the 27th quality control survey for immunoassay in Japan, 2005. Radioisotopes 55: 599-649 (In Japanese).

8. Subcommittee for Radioisotope in vitro Test, Medical Science and Pharmaceutical Committee, Japan Radioisotope Association, Immunoassay Research Society Japan (2007) A summary report on the 28th quality control survey for immunoassay in Japan, 2006. Radioisotopes 56: 637-686 (In Japanese).

9. Subcommittee for Radioisotope in vitro Test, Medical Science and Pharmaceutical Committee, Japan Radioisotope Association, Immunoassay Research Society Japan (2008) A summary report on the 29th quality control survey for immunoassay in Japan, 2007. Radioisotopes 57: 617-668 (In Japanese).

10. Subcommittee for Radioisotope in vitro Test, Medical Science and Pharmaceutical Committee, Japan Radioisotope Association, Immunoassay Research Society Japan (2009) A summary report on the 30th quality control survey for immunoassay in Japan, 2008. Radioisotopes 58: 655-708 (In Japanese).

11. Bidlingmaier M, Freda PU (2010) Measurement of human growth hormone by immunoassays: current status, unsolved problems and clinical consequences. Growth Horm IGF Res 20: 19-25.

12. Clemmons DR (2011) Consensus statement on the standardization and evaluation of growth hormone and insulin-like growth factor assays. Clin Chem 57: 555-559.

13. Wieringa GE, Sturgeon CM, Trainer PJ (2014) The harmonisation of growth hormone measurements: taking the next steps. Clin Chim Acta 432: 68-71. 